\title{
Diseño e implementación de soluciones sostenibles mediante una vivienda en la colonia Santa Clara, Comayaguela M.D.C. Honduras
}

\author{
Claudia Ester Bueso Andara ${ }^{1}$ \\ Irma Alejandra Valladares y Yesibel Osorto Lobo ${ }^{2}$ \\ Jorge $\mathrm{Corea}^{3}$
}

\section{RESUMEN}

Investigación de enfoque mixto basada en el diseño e implementación de técnicas y soluciones sostenibles, aplicadas a una vivienda prototipo con deficiencias cualitativas, en la colonia Santa Clara, ubicada en Comayagüela, del M.D.C, teniendo como objetivos identificar las condiciones espaciales y físicas de las viviendas existentes, y generar una propuesta basada en los resultados de la misma investigación que conlleve a la innovación y la fácil y adecuada implementación por parte de los habitantes de las técnicas de sostenibilidad de la vivienda. Para ello se tomó como referente un diagnóstico del lugar con herramientas de muestreo que permitieron la elección de las técnicas prioritarias, las cuales generen mejorar la calidad de vida de sus habitantes, que incluyan criterios de sostenibilidad, ecológicos, de bajo costo económico, de fácil realización con técnicas y materiales de la zona. De igual manera se consideraron temas de saneamiento básico, agua potable, seguridad alimentaria, manejo de desechos, reutilización del agua, y por supuesto las alternativas constructivas, permitiendo generar un resultado positivo en aspectos ambientales, económicos y sociales, sumando el uso de las técnicas e infraestructura implementadas por parte de sus usuarios. Basada en criterios de sostenibilidad, la investigación permitió demostrar a los habitantes los beneficios, accesibilidad económica y facilidad, con el fin de motivarlos a mejorar su calidad de vida con pocos recursos y materiales a su alcance. La vivienda podrá ser replicada las veces que sea necesario en la misma colonia.

Palabras clave: Arquitectura, sostenibilidad, vivienda básica, Honduras

\footnotetext{
${ }^{1}$ Beneficiaria de una beca de estudiante de grado, estudiante de Escuela de Arquitectura, Facultad de Humanidades y Artes, UNAH: buesoclaudia@hotmal.com

2 Estudiantes de Escuela de Arquitectura, Facultad de Humanidades y Artes, UNAH

${ }^{3}$ Profesor de la Escuela de Arquitectura, Facultad de Humanidades y Artes, UNAH
} 


\section{ABSTRACT}

The present study is a mixed approach research based on the design and implementation of sustainable techniques and solutions applied to a prototype dwelling with qualitative deficiencies in Colonia Santa Clara, located in Comayagüela, MDC, with the objective of identifying the spatial and physical conditions of the dwellings and generate a proposal based on the results of the same research that leads to innovation and the easy and adequate implementation by the inhabitants of the techniques of sustainability of housing. For this purpose, a diagnosis of the place was taken with sampling tools that allowed the choice of priority techniques, which lead to improve the quality of life of its inhabitants, which include criteria of sustainability, ecological, low economic cost, easy realization with techniques and materials of the zone. Likewise, issues of basic sanitation, drinking water, food safety, waste management, water reuse and, of course, constructive alternatives were considered, allowing a positive result in environmental, economic and social aspects, adding the use of techniques and infrastructure implemented by its users. Based on sustainability criteria, the research showed the inhabitants the benefits, economic accessibility and ease, in order to motivate them to improve their quality of life with few resources and materials at their disposal. The house can be replicated as many times as necessary in the same colony.

Key words: Architecture, sustainability, basic housing, Honduras 


\section{INTRODUCCIÓN}

La arquitectura y la construcción son actividades que contribuyen al desarrollo social y económico de un país. Problemas como la deficiencia y la falta de vivienda en la población generan un impacto en los medios económicos, sociales y ambientales; de igual manera el uso indiscriminado del espacio agota los recursos, altera el paisaje y aumenta la vulnerabilidad de riesgos naturales. Debemos enfocar nuestros esfuerzos para que nuestra sociedad logre una transición hacia un desarrollo sostenible, fomentado por medio de una propuesta arquitectónica atractiva, los efectos positivos que tendría a corto, mediano y largo plazo en el medio ambiente y en la interacción entre la ciudad y sociedad.

Para lograr esta transición podemos desarrollar y aplicar estrategias o técnicas, soluciones sostenibles, concretas y accesibles. Dichas estrategias deben buscar directamente la minimización de los impactos ambientales así como contribuir a la mejora de la calidad de vida de los usuarios y el entorno que los rodea, proponiendo con cada técnica ofrecer soluciones al manejo de agua, alimentos, control de desechos, mantenimiento de vivienda y saneamiento básico, por ser de las principales prioridades de la zona estudiada.

El interés principal de esta investigación fue analizar la situación actual de la vivienda hondureña, para impulsar un desarrollo sostenible desde la perspectiva arquitectónica, en una zona identificada de escasos recursos y de esta manera convertirla como un punto de partida para la implementación de estas nuevas tecnologías y así, de ser posible replicar los resultados en otras zonas similares y también en la construcción de nuevas zonas de infraestructura familiar. .

El campo de la arquitectura a través de la investigación y la búsqueda de un desarrollo sostenible, ha logrado generar una serie de propuestas de vivienda de tipo social para personas de escasos recursos. Dichas propuestas sirvieron como principios básicos para la implementación de las técnicas aplicadas, por medio de nuestro estudio integral de los factores psicológicos, materiales económicos y ecológicos, sumando otros aspectos como las condiciones climáticas de la zona, condiciones de asoleamiento y ventilación, también la re-utilización de materiales, y generación demecanismos accesibles de abastecimiento de servicios básicos. En fin se logra generar técnicas de sostenibilidad adaptadas a la zona y a las necesidades de los usuarios, y garantizar así un confort habitacional. 
Se puede observar que en los últimos años la intervención del medio ambiente a través de la arquitectura ha sido importante para el bienestar de la humanidad, sin embargo gran parte de nuestros problemas ambientales se deben a la intervención humana, esto debido a que centran sus actividades a determinados fines sin atender las múltiples consecuencias. La investigación y el desarrollo sostenible de la construcción deben orientar sus conocimientos a temáticas que generen principios que contribuyan a resolver problemas reales que enfrenta actualmente la calidad de vivienda en las áreas periurbanas de nuestro país y a la vez no dejar de atender las consecuencias no deseables de nuestros intentos por resolverlos, procurando así, la generación de una sociedad sostenible y comprometida con el medio que la rodea.

\section{METODOLOGÍA}

Se analizó la situación actual de la vivienda hondureña en zonas periférica de la ciudad denominadas como "de escasos recursos", con la finalidad de impulsar un desarrollo sostenible desde la perspectiva arquitectónica, generando un punto de partida para la implementación de estas nuevas tecnologías y así aplicar los resultados de ser posible, en otras zonas que presenten deficiencias habitacionales similares a la zona de estudio.

Nuestro tema focal gira alrededor de términos arquitectónicos que toman en cuenta el uso de técnicas de arquitectura sostenible, donde deseamos aclarar de manera técnica el significado de estos términos, incluyendo las técnicas que nacen a partir de este criterio que tienen como intención ser replicadas en el prototipo propuesto y ser la pauta para lograr las reproducciones en las demás viviendas que beneficiarían a los habitantes de manera integral. Según Brenda y Robert Vale, como se menciona en "La casa de autónoma" (Vale, 1978) la casa sostenible es más bien una nueva dirección a tomar por la sociedad, así estas técnicas benefician directamente a la sociedad que tanto necesita soluciones.

\section{DISEÑO}

La investigación se desarrolló bajo una metodología de enfoque mixto, ya que esta nos permitió la recolección y el análisis de datos cuantitativos y cualitativos, así como su integración conjunta, para realizar deducciones de producto de toda la información 
recabada y lograr un mayor entendimiento del fenómeno de estudio.

La aplicación de esta metodología en la comunidad de Santa Clara fue en base a la visión que tenemos de la búsqueda del bien común, en la cual se ejecutó una encuesta piloto para obtener información de los habitantes y los estados físicos de la vivienda, en dicho proceso se contó con la participación del comité del patronato; esta encuesta nos permitió ver la realidad del espacio y acontecimientos que se desarrollan en la respectiva área de estudio, lo que determino el desarrollo de las líneas de acción que se priorizaron.

Asimismo, para la recolección de información general de la comunidad, se visitaron instituciones específicas como el Instituto Nacional de Estadísticas (INE) y la información proporcionada por TECHO, en Honduras.

El objeto de estudio oriento hacia el tipo de diseños de investigación-acción el cual tiene por finalidad resolver problemas cotidianos e inmediatos y mejorar prácticas concretas. Por medio de este diseño de investigación se encontró información que guío la toma de decisiones para la aplicación de las técnicas de sostenibilidad, ya que ésta pretende esencialmente, "propiciar el cambio social, transformar la realidad y que las personas tomen conciencia de su papel en ese proceso de transformación". (Susy Méndez Pardo, 2006)

\section{POBLACIÓN}

La comunidad de Santa Clara se encuentra integrada por 400 viviendas divididas en 80 hogares. La población cuenta con 308 habitantes, de los cuales 67 son hombres, 91 son mujeres y 150 son niños. La población masculina en su mayoría conoce un oficio como albañilería, zapatería, carpintería o soldadura. Entre la población femenina, la actividad predominante es el cuidado del hogar.

Se realizó un grupo focal con los integrantes del patronato, este grupo nos ayudó a estructurar la herramienta investigativa a aplicar (encuesta), se seleccionó una muestra del $25 \%$ es decir 100 viviendas de la zona, la encuesta aplicada tuvo la finalidad de vincular la fase cualitativa, por lo cual se hicieron entrevistas no estructuradas, esto por medio de visitas personales a los moradores de las viviendas y así se logra la codificación de las expresiones de los encuestados con las distintas preguntas se obtiene una base de identificación de problemas y priorización de los mismos. 
Asimismo en cuanto a la fase cuantitativa la encuesta detalla la aplicación de una escala de materiales, asimismo contiene datos sobre las edades y el número de personas que habitan la vivienda visitada.

\section{Imagen 1. Vista de las viviendas colonia Santa Clara. Fuente: foto tomada por Fernando Amay}

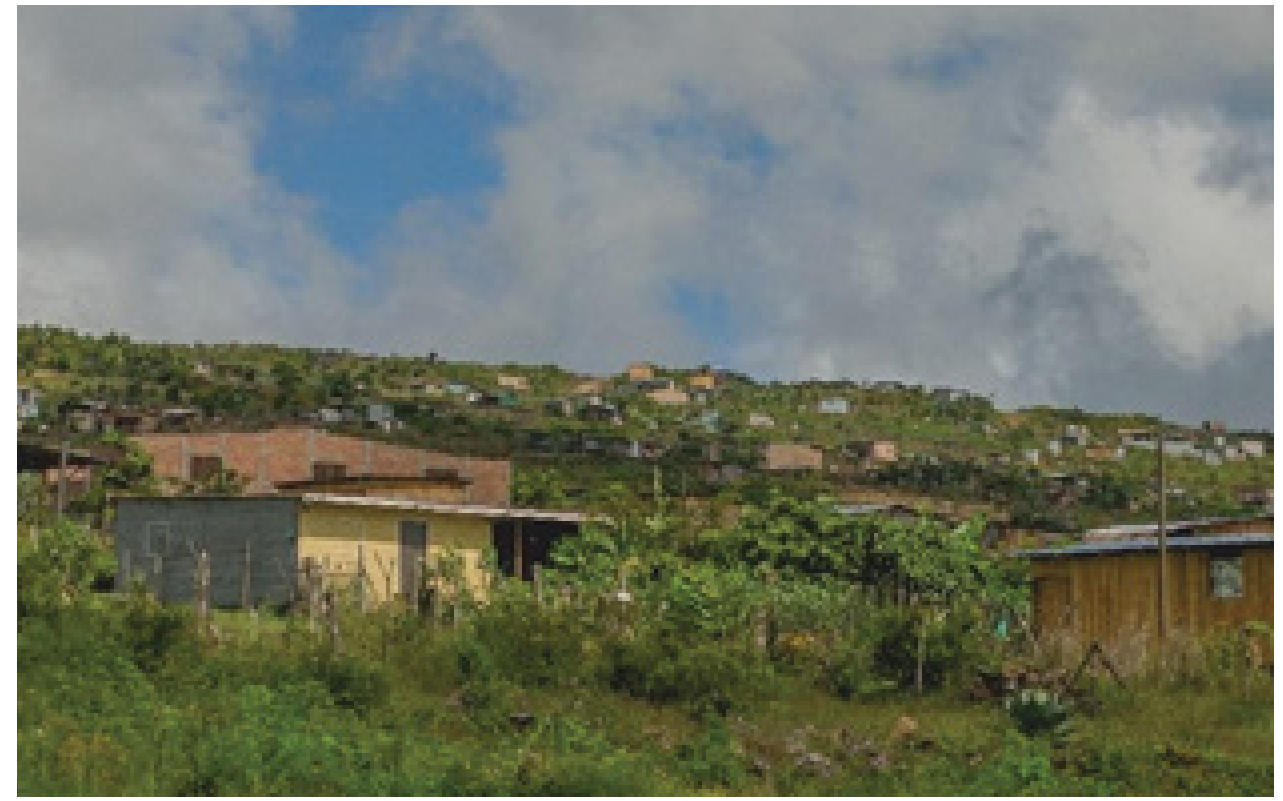

Fuente: foto tomada por Fernando Amay

\section{ENTORNO}

La Colonia Santa Clara se encuentra ubicada al nor-oeste de Tegucigalpa depto. de Francisco Morazán; para acceder a la comunidad existe una sola vía de ingreso, y para poder llegar hasta ella es a través de los accesos a nivel del anillo Periférico atravesando la Colonia Arturo Quezada y una calle de herradura a la altura de la Colonia Nueva Capital. Urbanísticamente, es una comunidad dispersa, con una división de 2200 lotes en los cuales solo residen 80 familias, razón por la cual se aprecia de manera esparcida y aislada; no cuenta con centros de salud ni de educación; existen tres iglesias evangélicas, además de un lote destinado para la futura construcción de una iglesia católica; La manera en que la comunidad ha establecido sus viviendas, han sido sin criterios arquitectónicos adecuados que consideren confort, condiciones adecuadas espaciales, o alguna orientación con el cuidado ambiental. 


\section{RESULTADOS}

La tabulación de las encuestas nos permitió completar nuestro objetivo de conocer las condiciones actuales de las viviendas de la colonia, reflejado como en el gráfico 1 y (Imagen $\mathrm{N}^{\circ} 2$ ) esto nos permitió conocer cuales técnicas de sostenibilidad eran prioritarias para los habitantes; además nos ayudó a definir cuál sería la vivienda que serviría como prototipo en la intervención y a la vez sirva de ejemplo para la aplicabilidad de cambios a los otros habitantes de la comunidad.(Imagen N.3)

Grafico 1. Resultados sobre el tipo de saneamiento utilizado resultado de las encuestas.Fuente: Diagramacion propia.

¿Qué tipo de aparato sanitario utiliza?

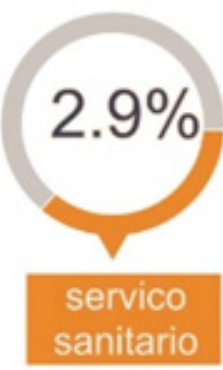

\section{letrina}

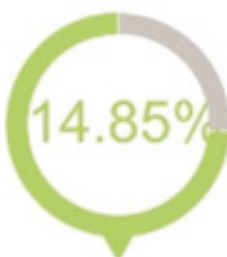

fosa septica
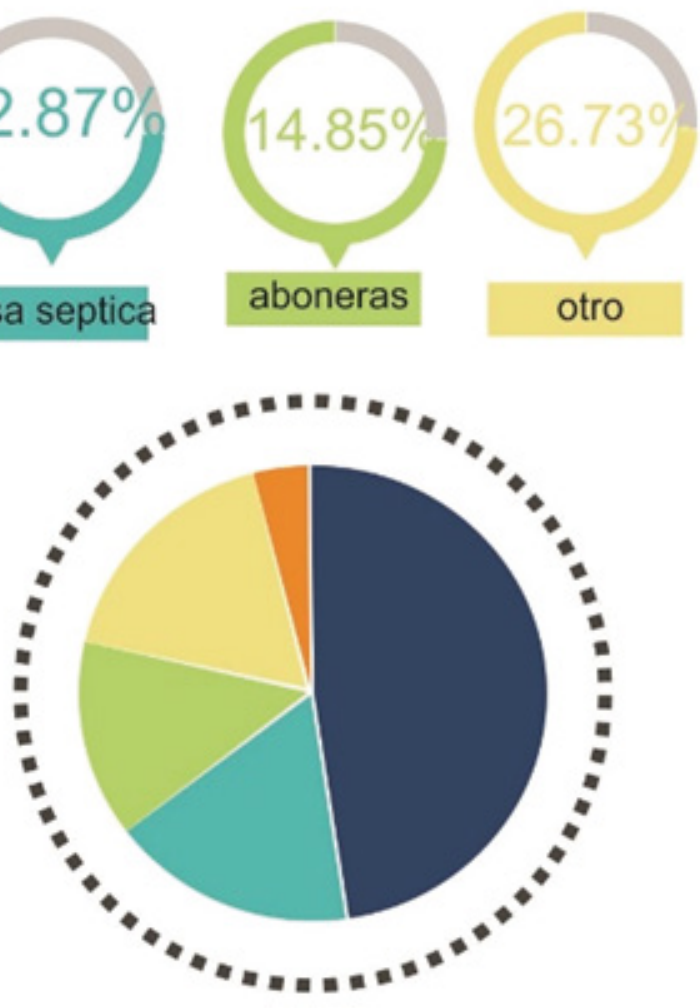

Fuente: Diagramacion propia. 
Imagen 2. Condiciones de la vivienda prototipo sujeta a la implementación de las técnicas de soluciones sostenibles

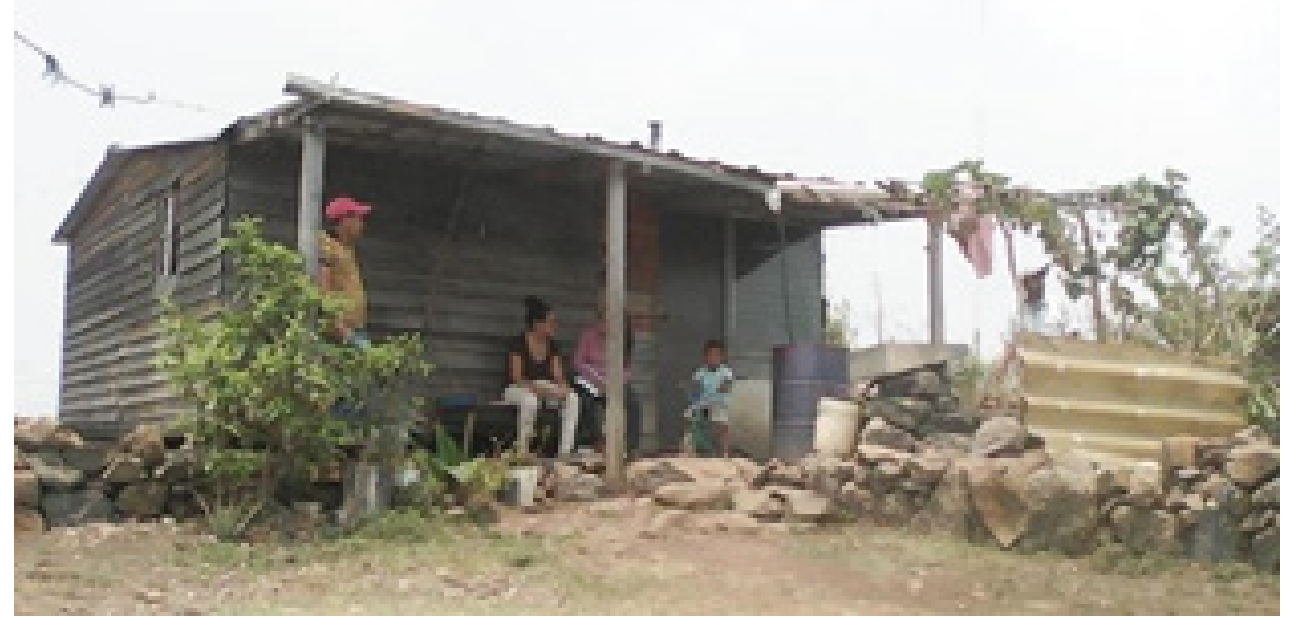

Fuente: Elaboración propia

Imagen 3. Ubicación y aplicación de técnicas en la vivienda
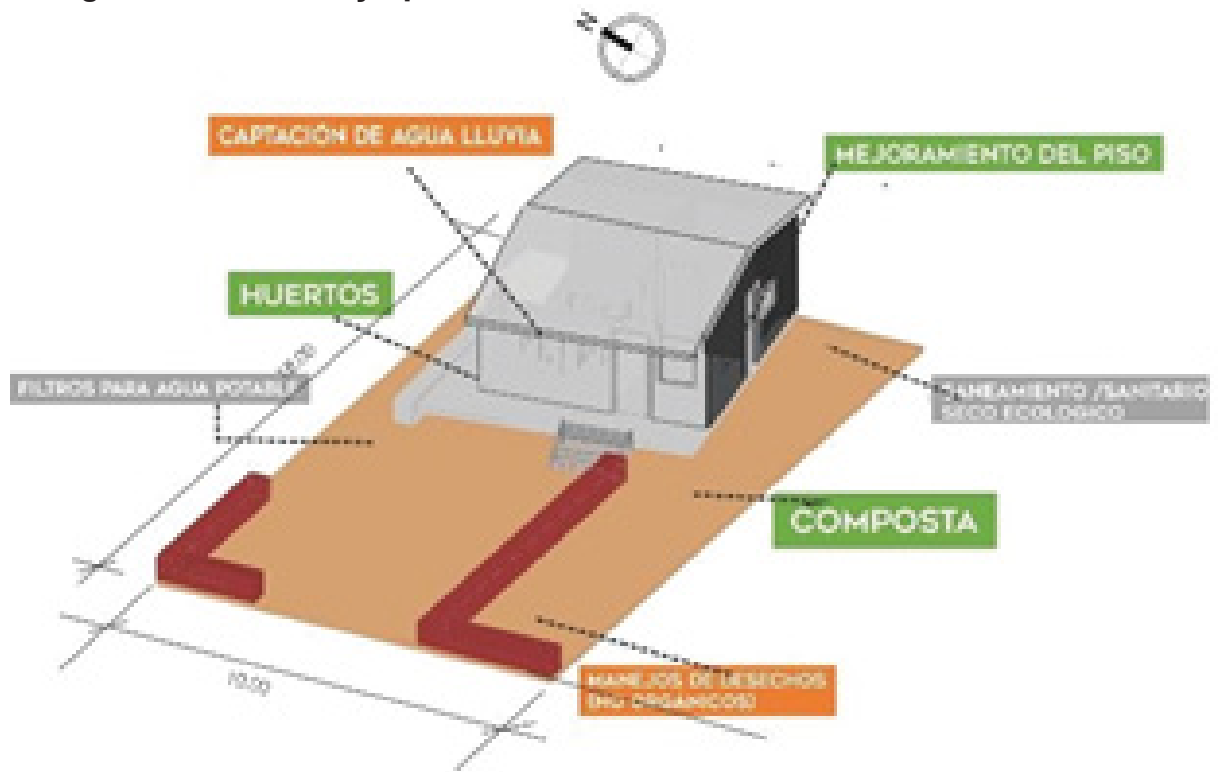

Fuente: Elaboración propia 


\section{Las técnicas aplicadas fueron las siguientes:}

1. Sistema de recolección de aguas lluvias, debido a la escasez de servicios básicos como el agua potable, la técnica viene a minimizar dicha escasez del vital líquido, pues permite la recolección durante la época de lluvia esto se logra por medio de la implementación de un tubo de PVC de 4" adosado al techo y dirigiendo el líquido por medio de un embudo que se anexa a un filtro de agua el cual termina en un tanque de almacenaje de agua. (Imagen $\mathrm{N}^{\circ} 4$ )

2. Filtros para agua lluvia y aguas grises: ya que el agua es fundamental para el uso diario del ser humano, se buscó una solución para poder reutilizar las aguas grises producida por las actividades como ser lavar ropa, lavar los utensilios de la cocina, la ducha y lavamanos; cuando no se filtra el agua lluvia, la misma todavía contiene microbios pues arrastra sedimento proveniente del techo. Esta agua resulta insalubre cuando los habitantes la utilizan de manera directa. El filtro de agua consiste en un recipiente de plástico perforado en el fondo al cual se le coloca una malla metálica sobre este (esta pues hablamos de la malla, no?) una capa de algodón, seguido (seguida, pues capa es femenino) de una capa de $7 \mathrm{~cm}$ de arena y una capa de $5 \mathrm{~cm}$ de grava.(Imagen No.5)

3. Soluciones de cultivo de hortalizas: como una manera apropiada de ayudar en la economía de los habitantes y hacer un complemento en su régimen alimenticio, se optó por dos sistemas de cultivo, el primero consiste en el sistema tradicional de crear arriates para cultivar debido al tipo de hortalizas y el segundo consistió en huerto vertical haciendo uso de materiales reciclables como ser las botellas plásticas, estas se entrelazaron unas con otras para crear una estructura auto soportante junto con sistema de riego por goteo.(Imagen $\left.\mathrm{N}^{\circ} 6\right)$.

4. Tratamiento de residuos: Utilizando la Composta, como un complemento de los huertos y para garantizar un manejo productivo de la basura orgánica producida en la vivienda, se planteó el uso del compostaje. el cual transforma estos desechos en humus o tierra vegetal, (Ecoinventos, 2016), dicha técnica consiste en la puesta de capas sobre capas de materiales orgánicos de la siguiente forma: la primera capa material seco (ramas y hojas secas) la segunda capa materiales húmedos (restos de poda y restos de cocina orgánicos) y una capa final de tierra combinada con estiércol para acelerar el proceso de tratamiento del residuo. (Imagen $\mathrm{N}^{\circ} 7$ )

Control de aguas negras: sanitario seco ecológico, (Imagen $N^{\circledR} 8$ ) la falta de servicios básicos en la colonia en particular el manejo de las aguas negras, nos orientó 
a priorizar el manejo de los desechos sólidos; de esta manera se concibe el uso del sanitario seco, pues así recibe los desechos en general y luego de un tiempo lo convierte en abono por medio de la descomposición solar, eliminando toda clase de patógenos. $(M, 2009)$. El sanitario seco ecológico genera un rendimiento óptimo, pues resulta que el depósito es bastante grande, el cual sirve para cubrir la demanda de uso de un núcleo familiar. Dentro de sus ventajas del uso del sanitario seco ecológico se encuentran las siguientes: en primer lugar soluciona el problema de eliminación de todos los desechos orgánicos, no hay que separar la orina, no necesita agua, es económico, produce abono constantemente a partir de los dos años, se usa material convencional para su construcción, en nuestro caso utilizamos pallets o tarimas de madera para construir las paredes, pedestales de lavamanos y letrina, también usamos piedra extraída del lugar y se utilizaron llantas de desecho para construir la cimentación.

\section{Imagen 4. Diseño y Concepto del Sanitario Seco ecológico.}

\section{SANEAMIENTO /SANITARIO SECO ECOLOGICO}
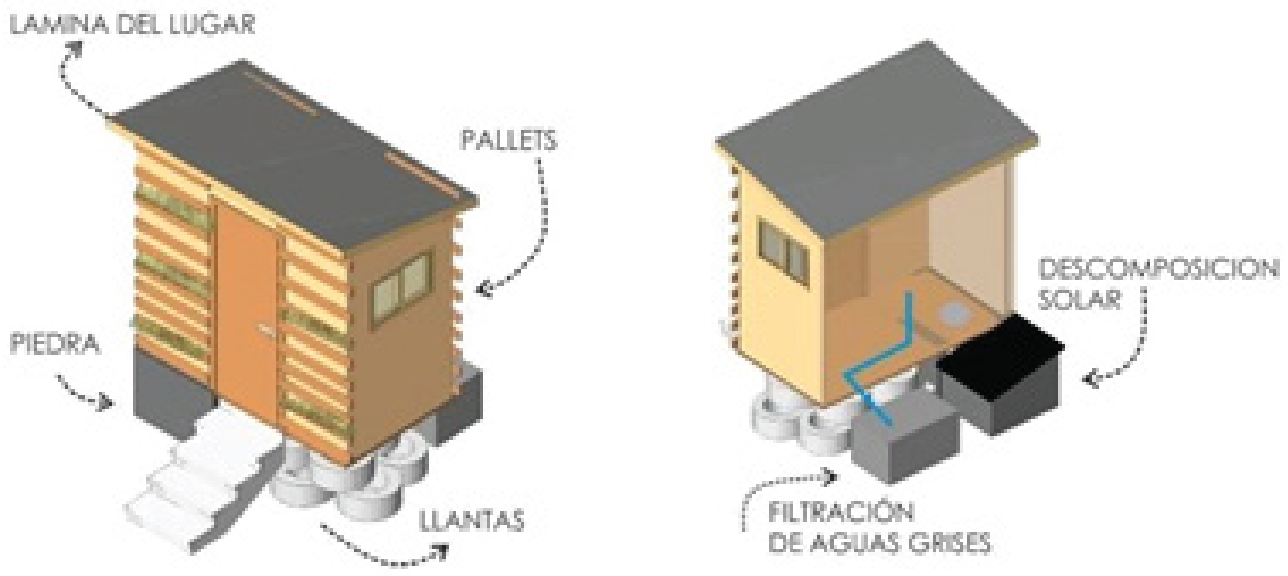

Fuente: Elaboración propia

5. Desinfección solar de agua con botellas: método SODIS (Short for Solar Water Disinfection) (Technology, 2011) La dificultad de los habitantes de la colonia para obtener agua potable es complicada debido a la ubicación geográfica del habitat, dado a que la misma colonia se asienta en una zona elevada por esta razón se optó por implementar un método fácil y seguro para sanear el agua y así mantener 
la salud de los usurarios, este proceso se denomina método solar de desinfección del agua (SODIS) es una tecnología simple usada para mejorar la calidad microbiológica del agua potable. SODIS utiliza la radiación solar para destruir los microorganismos patógenos que contiene el agua. El procedimiento es ideal para tratar cantidades pequeñas de agua. El agua contaminada se almacena en botellas plásticas transparentes y se expone a la luz del sol completa durante unas 6 horas mínimo. La luz del sol trata el agua contaminada a través de dos mecanismos sinérgicos: radiación en el espectro de la temperatura del agua a través de rayos UV (Iongitud de onda los 320-400) y aumentando la temperatura del agua. Si la temperatura del agua alcanza los $50^{\circ} \mathrm{C}$, el proceso de desinfección es tres veces más rápido y bastaría solo con una hora de sol. El envase necesita exponerse al sol durante 6 horas si el cielo es brillante, caso de estar 50\% nublado el envase necesitaría una exposición al sol de 2 días consecutivos, pues resulta que en los días nublados, la desinfección no se realiza satisfactoriamente. (Imagen $\mathrm{N}^{\circ}$ 9)

6. Piso ecológico, buscando la reducción del consumo de materiales no renovables se pretende estimular la reutilización y el reciclaje de materiales como llantas de desecho, botellas PET, entre otros, así se utilizan los materiales por metro cuadrado, generando un factor de disminución de costos en la endeble economía familiar . Esto trata de lograr construcciones cuyo ciclo de vida sea extenso, construyendo con calidad y a menor costo. El procedimiento es ideal y adaptable para implementarse en diversos lugares. Éste proceso se realiza preparando el terreno realizando la excavación de acuerdo al ancho de las llantas usadas (lo anterior es variable debido a la proporción del diámetro de la llanta), después se marcan las zonas en cada una de las llantas donde será perforadas para después continuar con la cocción las mismas y realizar así lograr un petate de llantas, una vez realizado el petate de llantas se inicia el relleno del terreno con tierra apisonada y piedras. Se coloca un firme de tierra apisonada (grosor variable de acuerdo a la altura del piso) (Imagen $\mathrm{N}^{\circ} 10$ ). Al final se coloca un acabo de barro con cal, ceniza, estiércol y arena cernida y una última capa de cal para terminar el pulido de la zona. (aldeas verdes, s.f.)

Las técnicas elegidas permitieron dar una solución a problemas como ser la falta de saneamiento, manejo de desechos, almacenamiento de agua potable, pisos inadecuados entre otros. Ver Imagen 5. 
Imagen 5. Resultados obtenidos en el proceso de diagnóstico, que dieron la pauta a las técnicas prioritarias aplicadas

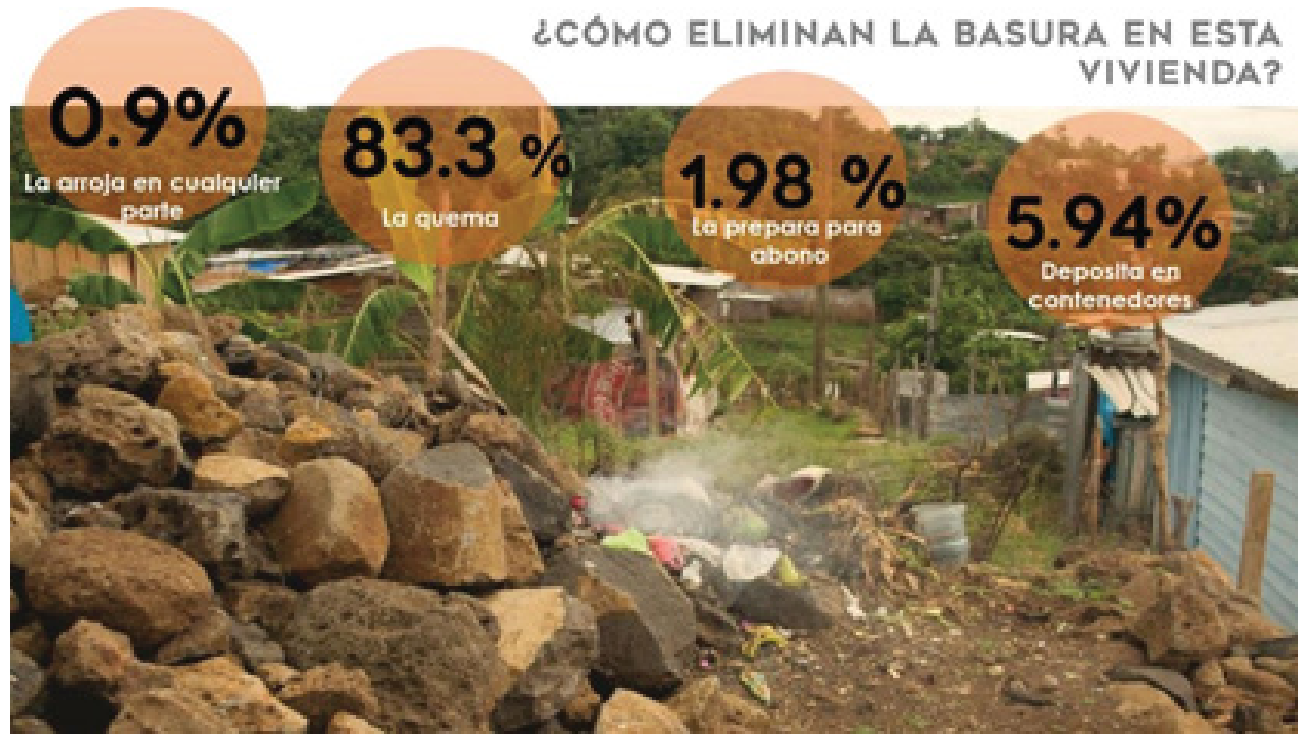

Fuente:Diagramacion Propia , Tabulacion de Datos de encuesta

\section{ANÁLISIS DE RESULTADOS}

Como resultados obtenidos de la aplicación de las técnicas, logramos analizar dichos resultados en los aspectos ambientales, económicos y sociales: En aspecto ambiental, la utilización de materiales del sector fue vital para aprovechar recursos, ya que habiendo analizado cuales fueron los materiales que más se desechan, diseñamos que técnicas elaborar en base a ello. Los plásticos son unos de los desperdicios de mayor consumo, y de mayor tiempo de descomposición, generando contaminación, posibles crías de animales y mal aspecto urbano.

La implementación de huertos verticales hechos con botellas, tienen el propósito de dar otras utilidades de mucho provecho al material. Además que debido a la falta de servicio de recolección de basura, se logra iniciar el uso del compostaje, basado en la creación de abono a partir de los desechos orgánicos, favoreciendo a disminuir la contaminación y acumulación de basura y favorecer a los cultivos producidos y por último se disminuye la contaminación ambiental, desde el momento que los usuarios 
tienen un espacio donde realizar sus necesidades fisiológicas, creando a partir de éste abono para un ciclo sostenible utilizando la energía solar. Uno de los problemas de la vivienda como de la comunidad, es la filtración aguas grises en el exterior por la falta de red de hidrosanitaria, pero debido a la implementación de filtros, ha permitido no contaminar más el suelo y reutilizar esta misma agua para otros usos.

Imagen 6. Recolección agua lluvia

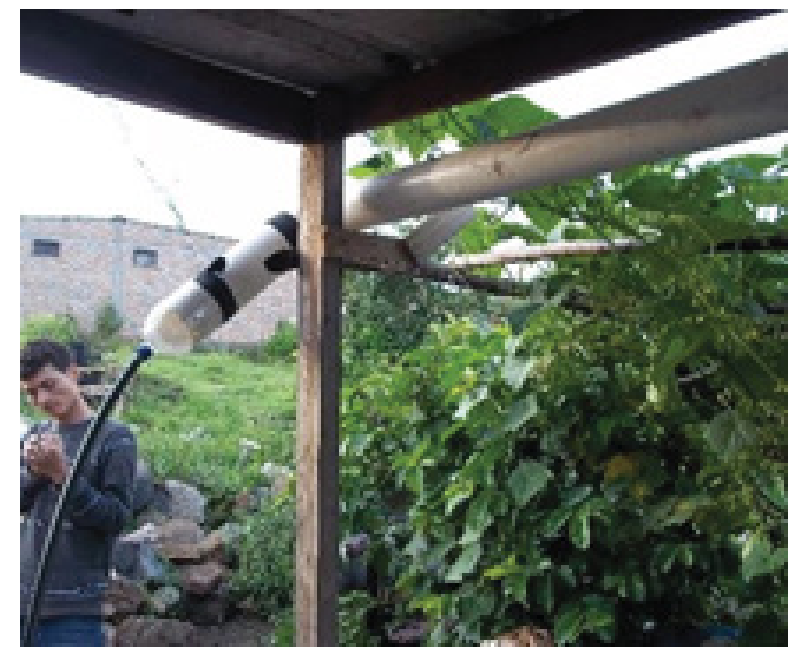

Fuente Propia

Imagen 7. Descomposición Solar y Filtros de aguas grises

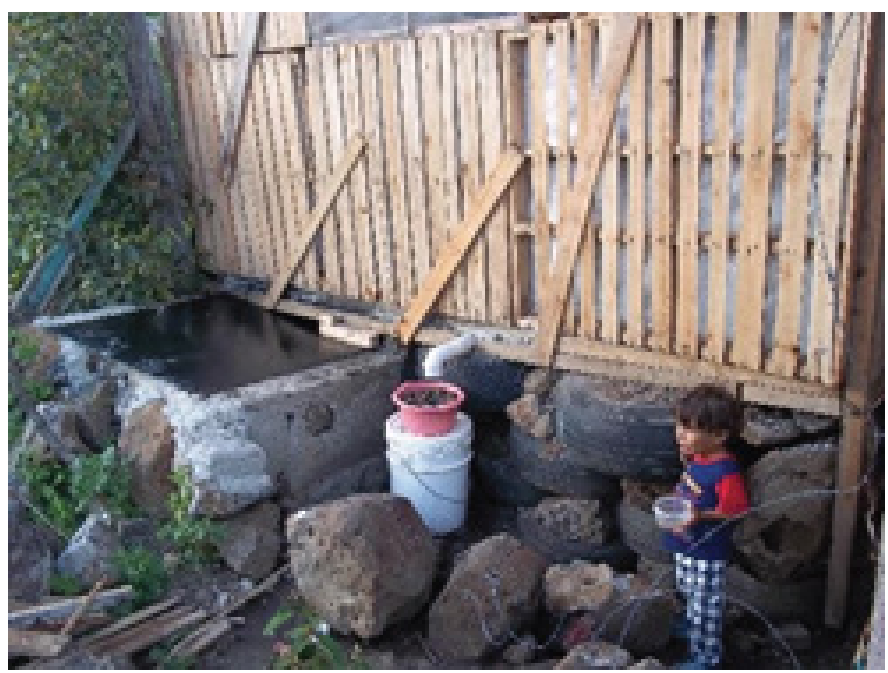

Fuente Propia 


\section{Imagen 8. Huertos Verticales}

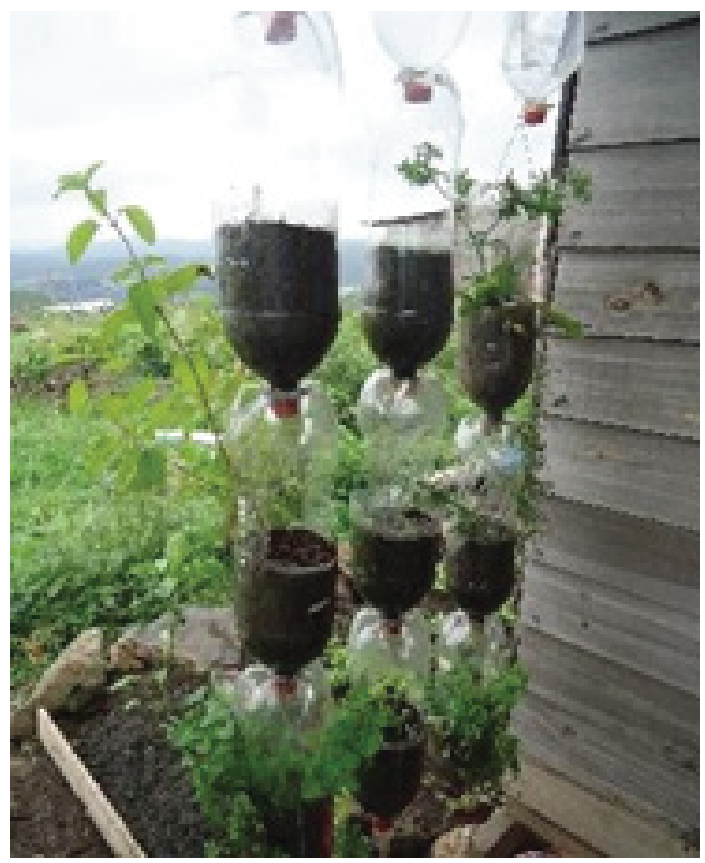

Fuente Propia

Imagen 9. Compostaje y huerto familiar

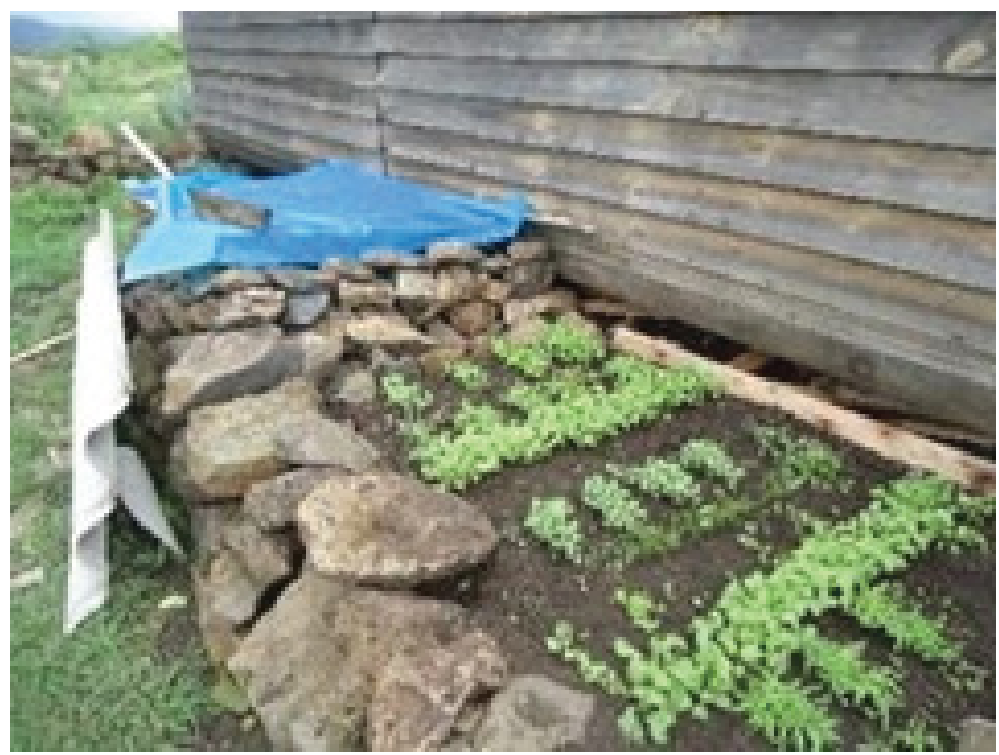

Fuente Propia 
Imagen 10. Detalles del interior del módulo saneamiento y fachada del módulo de saneamiento.
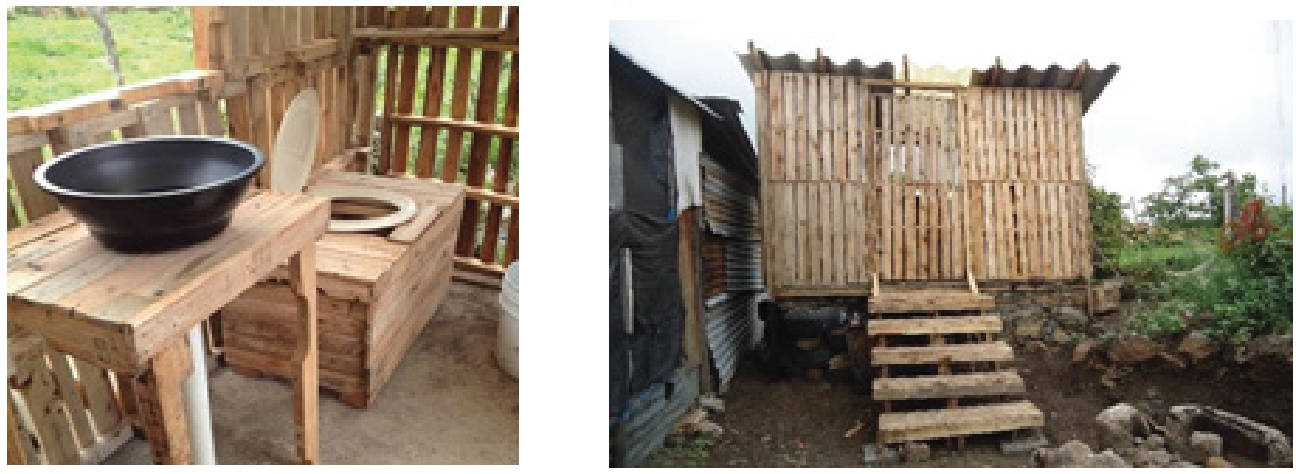

Fuente Propia

\section{Imagen 11. Método SODIS}

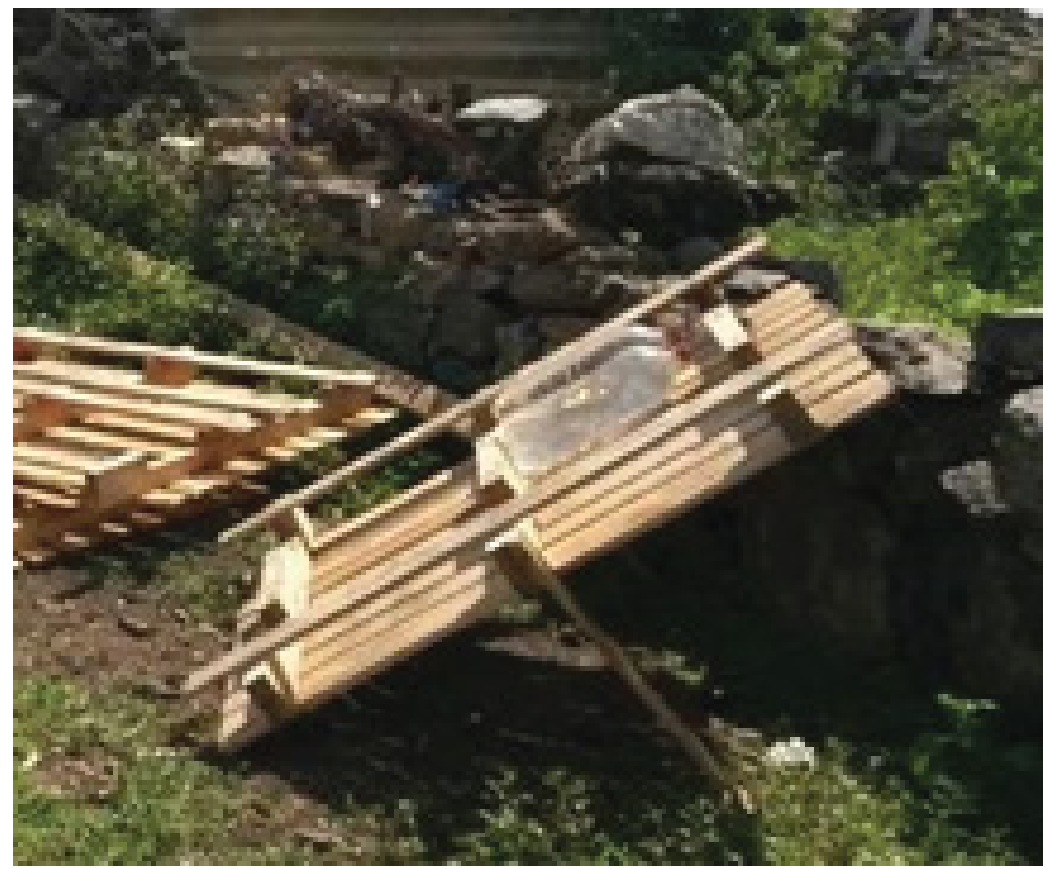

Fuente Propia 


\section{Imagen 12. Procedimiento del piso ecológico}

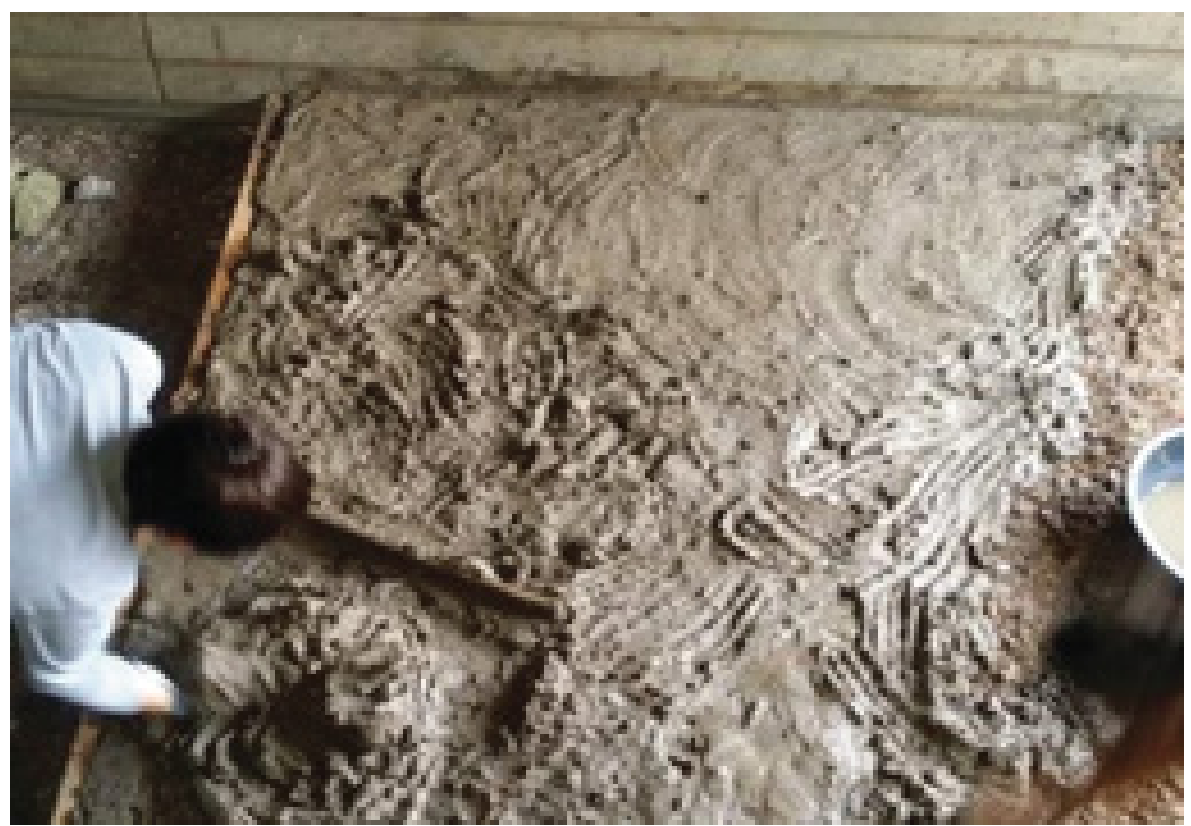

Fuente Propia

Imagen 13. Criterios de ventilación e iluminación

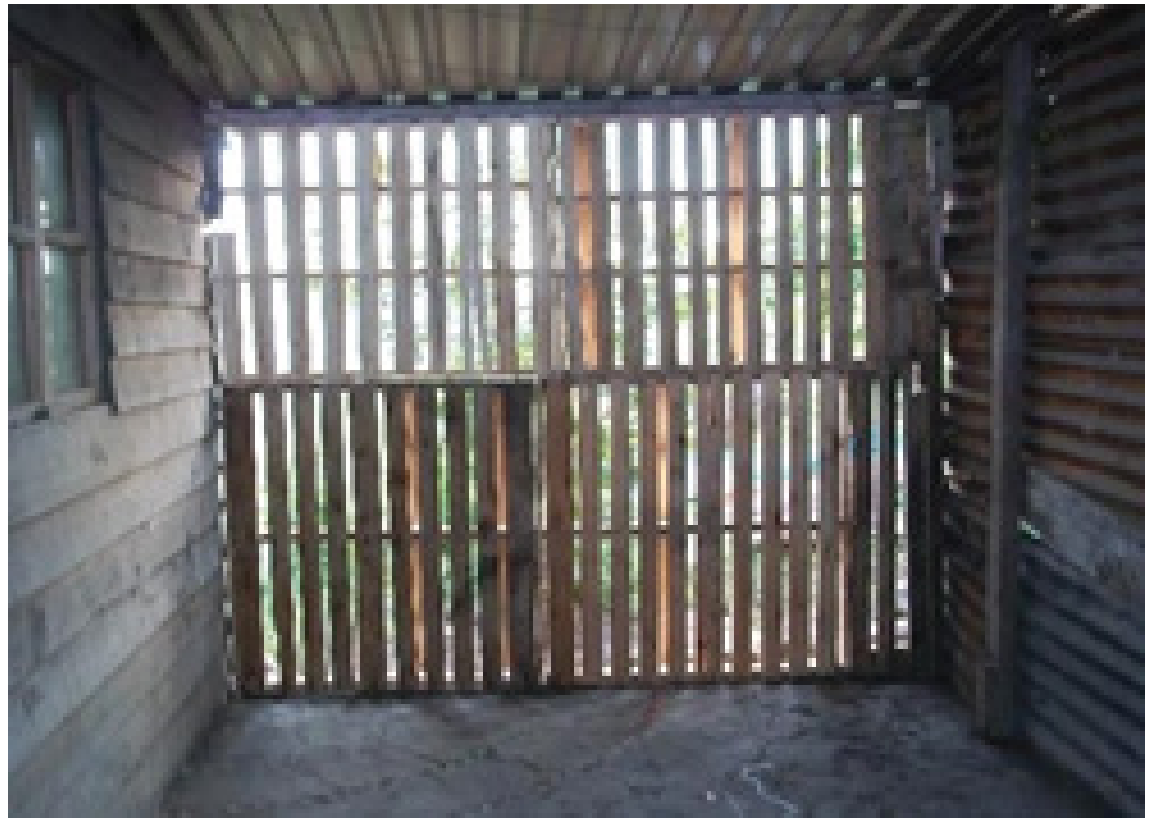

Fuente Propia 
En el aspecto económico, cada técnica se caracteriza por procurar gastar monetariamente lo menos posible en su obtención, y construir con un material de fácil acceso. La piedra, el barro y plásticos son materiales que abundan en la zona, todos los materiales fueron aprovechados para realización de las propuestas de construcción, disminuyendo considerablemente los costos. Dichos materiales, en el caso de las llantas y las botellas pueden encontrarse con facilidad en sitios similares, donde se desechan y la población en general desconoce el uso en la construcción o desconocen el nuevo uso que se le puede dar. Es por ello que inculcar el reciclaje en dicha comunidad podría generar una cultura de ahorro y de reutilización de materiales, de esta manera se logra hacer conciencia de los beneficios ambientales que conlleva el uso alternativo de los mismos materiales reciclables.

Se promovió la importancia de la economía en la familia de la vivienda, y esto se logró con la producción de sus propios alimentos e incluso como pueden llegar a una rentabilidad económica, al lograr abastecer la vianda de los moradores de la casa, al igual el ingreso familiar puede incrementarse con venta de las hortalizas producidas en las parcelas.

Solucionar el alto costo y accesibilidad al agua consumible, es vital, más cuando este importante líquido, difícilmente llega a la zona y cuando se adquiere es con unos altos costos económicos. Es así que se instruyó en el desarrollo del sencillo proceso de adquisión y potabilización del vital liquido.

Como resultado en el aspecto social, la manera en que se desarrollan las técnicas, lograron formar la conciencia conocimientos y capacidades para cambiar ciertos hábitos que de hecho resultan vitales su diario vivir, por ejemplo los moradores de la casa fueron los protagonistas en la mano de obra, forjando aptitudes para lograr construir e instruir a los demás para implementar las soluciones que mitiguen sus problemas de convivencia de manera sencilla, además se inculca por parte de los padres a los menores de la familia en seguir el ejemplo de vida cuando logren reproduciendo estos conocimientos.

Uno de los criterios que enfatizamos es la generación de réplicas de las técnicas a nivel de la colonia y las otras colonias adyacentes, demostrado por parte de los moradores una buena recepción cuando lograron observar las técnicas finalizadas. Esto les llamo la atención, para poder incluir el aspecto estético de la construcción en el futuro. 


\section{Imagen 14. Familia de la Vivienda Prototipo}

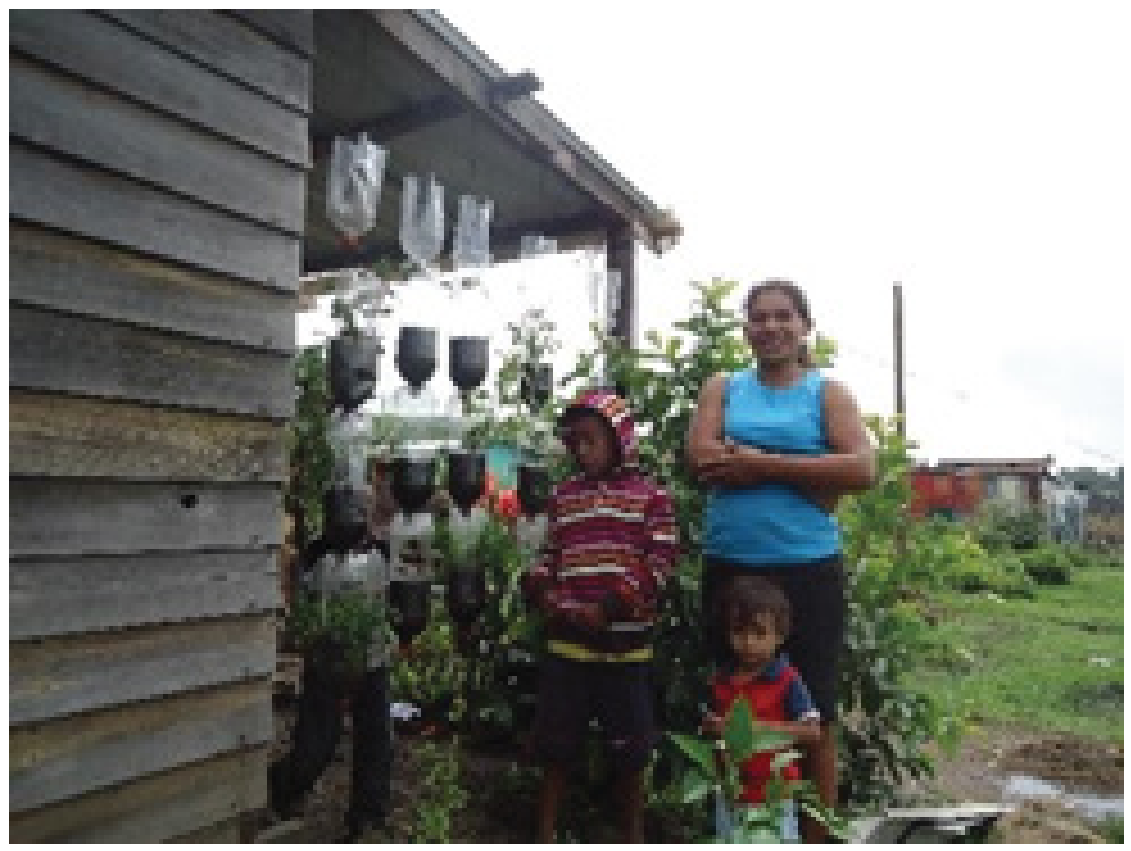

Fuente Propia

\section{Imagen 15. Participación en el proceso de implementación}

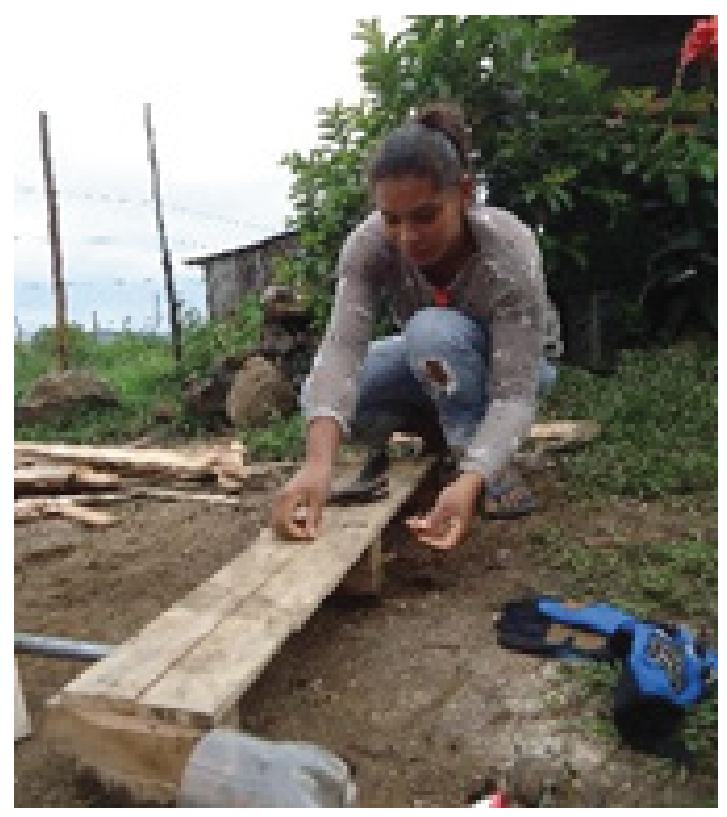

Fuente Propia 
Y para asegurarnos de orientar y promover la réplica del modelo implementado réplicas, se procedió a la elaboración del manual de las técnicas, lo cual tuvo un positivo impacto, cuando el manual fue leído como una guía sumando la visita al modelo de la vivienda, lo cual despertó las iniciativas de empezar a reproducirlas en sus respectivas comunidades.

\section{DISCUSIÓN}

Queda a discusión el tema de las nuevas maneras de valorar los criterios al proponer viviendas sociales, donde se descuida la sostenibilidad para su beneficio a corto y largo plazo, y sobre todo el acceso al conocimiento a soluciones accesibles a cualquier persona. Veremos cómo cada vez la arquitectura debe ofrecer soluciones al contexto que lo rodea, y llevar a aumento los resultados sociales, ambientales y económicos, que si llegan a alcanzar en gran escala, se favorecerán más personas ya que ellos mismos lo podrán hacer. Sin embargo limitantes como alternativas para su enseñanza a la población, resulta conflictivo si no se apoya de incentivos para la participación activa.

\section{CONCLUSIONES}

Finalizadas las etapas de investigación, el análisis de la comunidad, y el desarrollo de las técnicas de sostenibilidad en la vivienda prototipo y como punto final la entrega del manual con dichas técnicas implementadas se detalla las siguientes conclusiones:

1. Las condiciones espaciales de las viviendas demostraron tener altas deficiencias cualitativas, donde se comprobaban al mantener materiales de mala calidad, problemas de higiene con escaso o nulos sistemas de saneamiento básico, espacios de poca ventilación e iluminación, una difícil obtención de agua potable por la inexistente red hidrosanitaria, además de un inadecuado manejo de desechos.

2. La generación de una serie de técnicas basadas en las características específicas de la vivienda prototipo y de características de mayor coincidencia con gran parte de la comunidad, permitió demostrar a sus habitantes sus beneficios, accesibilidad económica y facilidad técnica; basados en criterios de sostenibilidad, incentivándolos a mejorar su calidad de vida con pocos recursos y materiales a su alcance. 
3. Se produjo un impacto positivo en la vida habitual de sus usuarios, desarrollando acciones de manejo más consciente de los recursos, cuidado de huertos como aspecto de productividad y dieta balanceada, una mejoría en el tema de salud con la construcción del módulo de baño, lavamanos y sanitario seco ecológico, y con la filtración de agua potable. Sumado a esto, se empezó a crear el hábito de utilizar los desechos orgánicos en el compostaje. Comprobamos como los usuarios de la vivienda prototipo explicaba de manera fluida a sus vecinos el funcionamiento y proceso de cada técnica utilizada, generando la publicidad de la misma por ellos mismos.

4. Nuestra experiencia de transportar materiales a la colonia nos hizo reflexionar y poner en evidencia la inaccesibilidad económica que resulta el pago de fletes a localidades retiradas, y es por ello que proponemos la necesidad de buscar alternativas constructivas con materiales de la zona y descentralizar el abastecimiento de materiales.

5. Las técnicas implementadas podrán generar soluciones a problemáticas no solo referentes a la Col. de Santa Clara, sino también a cualquier zona periurbana 0 urbana que incluya cualquiera de la problemáticas abordadas, siendo su uso a largo plazo de mayor beneficio económico, ambiental y de una cultura de sostenibilidad para la cuidad, accesible a todo tipo de habilidades y conocimientos.

\section{AGRADECIMIENTOS}

Deseamos agradecer el apoyo financiero de la Universidad Nacional Autónoma de Honduras y a la Dirección de Investigación Científica y Posgrado de la UNAH, DICYP, por apoyar a que se desarrollen este tipo de iniciativas. A la presidenta del patronato Margarita Cabrera de la colonia Santa Clara, por cooperar con mucho entusiasmo en distintas labores y procesos, los cuales fueron de mucha ayuda y además se extiende nuestro agradecimiento a todas las personas voluntarias que se unieron al desarrollo de esta investigación con un total apoyo de forma desinteresada lo cual contribuyo profundamente al éxito de esta investigación 


\section{REFERENCIAS}

Aldeas verdes. (s.f.). Obtenido de: Permacultura-ecologia-desarrollo sustentable: https://aldeasverdes.wordpress.com/

Ecoinventos. (2016). Obtenido de: Portal Fruticula: http://www.portalfruticola.com/noticias/2016/04/04/guia-para-elaboracion-de-compost/

M, W. U.-H. (2009). Saneamiento ecologico-edicion corregida y aumentada. Ciudad de México: Editorial Pax Mexico

Technology, S. F. (2011). SODIS. Suiza, Suecia: Ewag Research.

Vale, B. y. (1978). La casa autónoma, diseño y planificación para autosuficiencia. Barcelona: Gustavo Gili,S.A. 